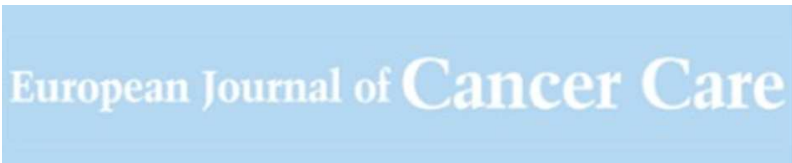

\title{
Undergoing Head and Neck cancer surgery: a Grounded Theory
}

\begin{tabular}{|r|l|}
\hline Journal: & European Journal of Cancer Care \\
\hline Manuscript ID & Draft \\
\hline Manuscript Type: & Original Article \\
\hline Keywords: & $\begin{array}{l}\text { Head and neck cancer, Grounded theory, Surgery, Decision Making, Patient } \\
\text { participation, Preoperative care }\end{array}$ \\
\hline \multicolumn{2}{|l}{} \\
\hline
\end{tabular}

\section{SCHOLARONE \\ Manuscripts}




\title{
TITLE
}

\section{Undergoing Head and Neck cancer surgery: a Grounded Theory}

\begin{abstract}
Objective: Surgery is the treatment of choice in most head and neck cancers. Very often, the surgery is demolitive with high impact on the psycho-social, functional and aesthetic fields.

Methods: We conducted a grounded theory study with semi-structured interviews to explore the psycho-social process occurring when a patient with head and neck cancer needs to undergo surgery.

Results: Seventeen participants (six patients, nine health care professionals, and two volunteers) were interviewed immediately before surgery. The study generated a process of "persuading the patient of an obligation" as the core category. The other principal categories that emerged highlighted the patients' doubts and fears regarding the surgery consequences and, in parallel, strategies employed by the health care professionals to contrast hindering issues impeding surgery. In particular, healthcare professionals involved patients in an affiliation process through simplified communication to sustain the choice of surgery; the family plays a supportive role in this process.

Conclusion: The interplay between the organizational process and patients' experience results in "I will let you convince me" at the end of the decision making process, where the main aim was to save and be saved.
\end{abstract}

Keywords: Head and neck cancer, Grounded theory, Surgery, Decision Making, Patient participation, Preoperative care 


\section{INTRODUCTION}

Head and neck cancer (HNC) refers to a group of tumours that develop in the body region of the head and neck and may affect the oral cavity, pharynx, larynx, nasal cavities, paranasal sinuses, and salivary glands. The incidence is estimated to be above half a million cases per year worldwide and represents, by diffusion, the fifth most common type of cancer (Harrison et al., 2009). The main factors that influence its development are alcohol and tobacco abuse (UK CCR, 2011). Due to the development of diagnostic-therapeutic techniques, the overall survival rate for this group of malignancies has slightly improved over the past decade, particularly for individuals with the highest socio-economic level (UK CCR, 2011). Most HNC cases are surgically treated, and their outcomes are often demolitive (Adelstein et al., 2017). Similar to other oncological diseases, the diagnosis of an HNC has a significant psychosocial impact on patients (Chaturvedi et al., 1996) who may experience uncertainty about the consequences of surgery on their physical, functional and psychological well-being (Hutton \& Williams, 2001). The facial region is an important aspect of personal identity, and changes in its image often cause intense suffering and embarrassment (Macgregor, 1990; Fingeret et al., 2012). Moreover, many patients have to face significant changes in functions such as communication, breathing, swallowing and the sense of taste (Harrison et al., 2009; Howren et al., 2013). In particular, the disfigurement and impairment that often result from surgical treatment may emerge as stigmatization (Macgregor, 1990) because patients may blame themselves for their risky behaviours (e.g., smoking and alcohol abuse) (Fife \& Wright, 2000).

From $15 \%$ to $50 \%$ of patients report clinically relevant anxiety and depression (Haisfield-Wolfe et al., 2009; Llewellyn et al., 2005; Threader \& McCormack, 2016). Most studies on the psychosocial aspects of HNC are quantitative. They have highlighted the prevalence of anxiety and depression in this population and the relationship between these symptoms and coping skills, quality of life and other variables related to treatment outcomes (Dropkin, 2001; Horney et al., 2011; Moore et al., 2014). Only recently have these aspects also been studied using qualitative approaches. These studies primarily investigate the consequences of surgical treatment (Lang et al., 2013), while the 
understanding of how patients experience the pre-surgery phase is underdeveloped. The few qualitative contributions that discuss the pre-surgical phase mostly address the topic of information/communication related to treatment and possible consequences (Ragaccio et al., 2007; Sharloo et al., 2005). Information received in the preoperative phase was often provided too quickly and was perceived as too detailed and too complicated for the emotional status of the patients, thus affecting their decision-making process (Ragaccio et al., 2007; Sharloo et al., 2005). This incomplete awareness could adversely affect the patient's coping strategies and prognosis (Sharloo et al., 2005), as also reported in different oncological populations (Mesters et al., 2001; Van Der Molen, 1999).

A grounded theory (GT) study (Konrades et al., 2009) revealed how profoundly impacting issues, such as disfigurement, are not addressed appropriately in the literature. Dropkin (2001) showed that when health professionals anticipate the disfigurement issues before surgery, patients would respond by developing high levels of anxiety and lower coping abilities. On the other hand, a high level of uncertainty about surgical consequences would adversely affect the prognosis (De Boer et al., 1998).

Based on current knowledge, no study has focused on the HNC patients' perspectives in the presurgical phase.

Understanding the patients' experience from the diagnosis to the surgical treatment would be beneficial to improve the quality of pathways considering their practical, psychological, and relational needs. This study was aimed to explore the psycho-social process that occurs when a patient with $\mathrm{HNC}$ cancer receives communication about the need to undergo surgery. Our research question is as follows: "what is going on when healthcare professionals propose surgery to an HNC patient?" 


\section{METHODS}

\section{Methodological framework}

To address the research question, we followed the Charmaz's constructivist grounded theory approach (GT) (2006). GT is a general method of interpretative research adopted by social scientists to define the processes underlying interpersonal interactions (Charmaz, 2006; Glaser, 1978). We adopted GT to develop a theoretical model that explains which factors may influence the interpersonal process and which challenges patients, their families and the HCP are facing.

\section{Setting and sampling}

The GT study was carried out at the otolaryngology ward of the XXXXXXXXX. The researchers identified two additional contexts for the research: the XXXXXXX and the XXXXXXX.

\section{Inclusion criteria}

The HNC patient candidate for surgery and his/her family, caregivers and HCP represented the unit of analysis of this study.

Our sample included the following:

- adults diagnosed with HNC, waiting for surgical treatment

- family members and/or caregivers considered significant by the patient

- subjects who comprehend and speak Italian

- HCPs who work in clinical settings involved in this research

\section{Sampling procedures}

The sampling followed three steps: initial sampling, snowball sampling, and theoretical sampling (Charmaz, 2006; Bagnasco, 2015). The initial sampling was purposive (programmed in advance) based on the research question. It involved four HNC patients, candidates for demolitive surgery, 
and three HCPs.

Thereafter, we applied snowball sampling where individuals already recruited indicated further potential participants (caregivers or HCPs) who played a significant role for them. At the end of this step, we included two more people, an HCP and a volunteer. During the data analysis, we applied the theoretical sampling to confirm and saturate the provisional categories involving two HNC patients, one volunteer and six additional HCPs.

\section{Data collection}

The E.L. XXXXX, M.G. XXXXXX, M.C.B. XXXXXX, and S.C. XXXXXXX collected data between May 2016 and January 2017. All researchers were female, had no professional relationship with the patients and did not work in the wards where the data were collected. They received one year of training in qualitative research methodology. When possible, the interviewer was introduced face-to face with the patient by the head nurse. All the interviews were performed in an appropriate place in the clinic (workplace for HCP) by an interviewer and an observer. Prior to the beginning of the interview, researchers explained to the participants the aim of the study that is, the need to deeply understand the process triggered when a patient is told the necessity to undergo surgery to remove a cancer in the head and neck region. Three semi-structured open-ended interviews were prepared: one for the HNC patients, one for persons indicated by the patients (PIP), and one for the HCPs (Table 1). Questions were asked in different orders, and themes were treated according to the priorities of the respondents. The interviewers explored all the key themes, posing clarification questions and exemplification requests. Additionally, the interviewer asked the participants about the opportunity to meet a second time in the case of doubts in the interpretation of the collected data. The researchers collected demographic information, qualitative data and field notes concerning nonverbal behaviours of the participants.

The interviews lasted between 20 and 60 minutes. The researchers audio recorded and transcribed verbatim the interviews within 48 hours from their completion. The participants were given the opportunity to read the transcript if requested. One patient asked to read the interview and 
confirmed the content.

\section{Rigor}

Two researchers collected the data on the field, whether the other two researchers played a role in the audit, checking the interview transcripts and coding. The four researchers who collected the data also open-coded the interview transcription line by line using the participants' words. The credibility of the explanatory model was pursued by collecting both in-depth data and field notes.

Moreover, the authors critically discussed and verified the relevance of the levels of coding, coherence of categorization made during focused coding and data saturation (Lincoln \& Guba, 1995; Finley, 2002).

\section{Data analysis and saturation}

The researchers started data analysis concurrently with data collection, following the GT indications for coding (Charmaz, 2006):

1. Open coding: researchers who conducted the interviews indexed them using codes and then shared the data with colleagues.

2. Focused coding: researchers grouped the codes into conceptual categories, identifying concepts at a higher level of abstraction. The first conceptualization was derived from the data of the first seven interviews from which the researchers identified nine conceptual categories.

3. Theoretical coding: researchers defined the explanatory theoretical model, highlighting the relationships between the conceptual categories. This phase has allowed for the data to be summarized in one core category plus three principal categories and their related subcategories. Researchers performed the theoretical coding in group under the supervision of L.G. and S.D.L. as external auditors. They stopped recruiting participants when the analysis reached data saturation: the latest interviews revealed no new information or insights. 


\section{Ethical considerations}

The Provincial Ethics Committee of XXXXXX approved the study (Protocol n. 2013/0009390 of 2013/04/09). The research was conducted following ICH E6 Guidelines for the GCP and the principles of the Declaration of Helsinki.

The study and its report followed the consolidated criteria for explicit and comprehensive reporting of qualitative research checklist (Tong et al., 2007)

\section{RESULTS}

The final sample included 17 participants (six patients, nine HCPs and two volunteers) whose characteristics are reported in Table 2. Researchers conducted 18 interviews: P6 was interviewed twice to clarify some doubts and gather more information. During the interviews, only patients and researchers were present. In one case, the patient's wife was present for half of the interview duration (P3). HNC patients did not indicate any PIP.

\section{Explanatory theoretical model}

Based on the conceptual categories emerging from the data, we developed an interpretative model to answer the research question, "What is going on when healthcare professionals propose surgery to an HNC patient?". The theoretical model develops through three phases and explains the psychosocial process that occurs when a patient with $\mathrm{HNC}$ is receiving communication about the need to undergo surgery (Figure 1).

\section{Core category: persuading the patient of an obligation}

What happens in the context studied is a process where HCPs employ strategies to convince the patient to accept the surgery, and conversely, the patients allow themselves to be convinced. What we call "persuading the patient of an obligation" is the core category, which connects all the 
categories into a conceptual framework. The term "persuading" is used in the sense of "influence to act, to make a decision". In our data, the pivotal concept is surgery seen as an obligation.

"I told him [to the patient] that the most appropriate therapy for the disease according to international guidelines is the surgery... You have to do" (HCP1).

"I told her: you have a malignant laryngeal cancer, and we have two options: one is radiotherapy, and the other is surgery. We decided to do surgery" (HCP5).

"Because... there are no alternatives... there is nothing to do. So, I calmed my heart down, and I accept what comes" (P4).

\section{Description of principal category: interferences}

The process of "persuading the patient of an obligation" begins with a first phase in which some impeding factors have been identified as "interferences". Those factors are manifold and concern the following: 1) shock for the diagnosis experienced by the patients; 2) patients' expectations regarding the functional outcomes of the surgery; 3) cognitive and educational levels of the patients; and 4) professionals who potentially might be "offline".

1) Patients receive a diagnosis of cancer and almost immediately the proposal for surgical treatment. Patients are very scared.

"It is going to be bad... what do you want me to say? [palms to the ceiling] I would throw myself out of the window" (P5).

"I am afraid I cannot... I have a throat cancer... and I am desperate..." (P1).

2) The expectations regarding the functional outcomes of the surgery may delay the acceptance of the surgical procedures: the patients' attention seems to be focused on the life-changing adaptations, regarding the voice, speech, and swallowing changes, rather than on the cancer.

"Well... my fear is to totally change my life, in the sense that... for what my doctor anticipated... I will have to change both the way I eat and the way I talk... so... a fear... remarkable that I have because I do not know when I will wake up... under what conditions" 
(P3).

"They [the patients] mystified and try to assume an attitude as if it was not the disease to be solved but the voice problem, so they ask if there will be a way to talk and feed... without facing the 'cancer' topic" (HCP1).

"...they [the changes] are difficult to accept, are life-changing. They change the lives of family members, in many ways, not only from the point of view of the voice, speech, eating and swallowing, but in the very intimate sphere of relationships with the family, with the wife, husband..." (HCP6).

3) The cognitive and educational levels of patients may influence the acceptance of surgery.

"When patients have a certain type of high or medium-high schooling, or have an activity where voice is crucial... here, it becomes difficult because the voice becomes dominant... they see their world collapsing... and then other difficulties when they are informed from the internet. They believe they know everything, and then... say what they want you to do without having the awareness of the problem... For less educated people, the word 'cancer' is still like a divine curse, so they have the concept of saving the skin. I am the one who saves their skin" (HCP1).

4) The presence of professionals who might be "offline" can interfere with the need to persuade patients of the obligation. The "offline" professionals are the newly hired with little familiarity with HNC patients or those considered as outside the "culture" of the otolaryngology ward.

"The surgical decision does not involve us or at least not in this hospital, not in this type of management and... I have never been summoned to the tumour board" (HCP6).

"What helps us is the fact that we are all tenure nurses, and we have worked together for many years... we all have the same way of managing and experiencing these situations... The new staff does not deal with these types of patients. A model has been created... we all do the same thing... those who came after... at the beginning... have a different way of managing... but after a while, they do as we do" (HCP2). 


\section{Description of principal category: contrasting hindering issues}

In this phase, the interferences to surgery adherence are managed by a twofold strategy, which includes affiliation and neutralization attempts. Furthermore, the family may play an important role in contrasting issues that could delay surgery.

Regarding the affiliation attempt, data analysis showed that HCPs act within a departmental culture and build a unified and shared communication modality. HCPs are very confident about themselves and their way of working.

"When we have a patient to whom we have to do some kind of intervention or tell him about a diagnosis, we always do it collegially. Let us first talk about it and decide together what to say, and then... we share the same attitude and keep the same version" (HCP1).

"Beyond standardized procedures, we have adopted a behaviour... this happens daily because these are the patients we see for a long time. They are so specific that they can only be managed here, with our features" (HCP2).

The HCPs are committed to build a departmental culture in which we feel like a family and tend to define patients as "our" patients:

"When a family relationship is established, we are their family" (HCP4).

Furthermore, these strategies contribute to gain total trust from the patients.

"I trust the professor. I sought information from other doctors, but they earned my trust here. The doctor said he will do all he can. They [the HCPs] have studied a lot! I gave him a white paper. Even if he does something that is not good, if it is necessary, he needs to do it. Even if there are two or three ways, I told him 'you choose the best'. If it is possible, I do not want the hole. However, they do what they can" (P2).

"I trust here, and that means so much" (P3).

The neutralization attempt explains how patients and HCPs justify surgery and adhere to it. HCPs 
and patients seem to agree in the stereotypical explanation of the pathology, while HCPs stigmatize the idea of HNC patients and patients blame themselves for the disease.

"Our patients are, in the vast majority, marginalized and... on the margins of society, in the sense that they are people who drink and smoke... people who have a disorderly life, especially in terms of smoking and alcohol consumption" (HCP1).

"It is my fault, I was smoking... it depends on smoke... if I tell you everything I did...Therefore, I was looking for it" (P1).

"If I had stopped drinking and smoking... I had been following the line, probably it [the cancer] would not jump out. Now I am paying for it. You are the one who wanted it. This is the truth" (P2).

The communication is simplified to reassure patients and provide them with general information. HCPs minimize potential side effects of surgery to neutralize fear and shock from the patients. They give them no specific information about foreseeable future conditions and delay all possible explanations to the postoperative phase.

"They are very frightened at first. What will happen is not clear to them because they have not understood what it means. In the days to follow [after the surgery], as soon as the situation is calmed down, when they realize that life continues, we will accompany them. We will explain things as if we were explaining to a child" (HCP2).

Sometimes, the possible consequences of surgery are minimized:

"I say to the relatives... 'You do not have to feel sorry for him. This is still good [touching the head], we miss the voice, but we have the head. You have to live the life you had before... going to the café, reading the newspaper, eating fruits, the meat" (V1).

The role of the family is important in persuading the patients to undergo surgery, supporting the team decisions and taking care of the emotional needs of their beloved.

"We try to figure out who the person is [the patient]... if we have to first approach the family to get to him" (HCP1). 


\begin{abstract}
"The situation depends very much on the family climate. If there are patients followed by relatives, giving support, it would be easier. All that can be a support, an incitement, an exhortation from the family helps" (HCP6).

"My family, honestly, for me... they support me, and they tell me 'you will see... everything will be put in place' " (P3).
\end{abstract}

\title{
Description of principal category: "I will let you convince me"
}

The process we studied ends with the patient's adherence to the proposal. If the affiliation and neutralization attempts work, the patients allow themselves to be persuaded to undergo surgery, representing "salvation" for all the participants.

"I have to do it. It is in my mind. If you want to live, you have to do it" (P4).

"And now we have to do this. I decided... to do... to live as I will can" (P3).

\section{DISCUSSION}

All the main categories emerged, converge on persuading the patient that surgery is a "salvation", and as a result, persuading the patient to undergo surgery is the core category. The process of persuasion and being persuaded develops within a patient-HCP relationship dominated by the need to have surgery to save and be saved.

The conceptual model developed explains the interplay between the organizational process and patients' experience. In fact, the HCPs activate the therapeutic pathway necessary to "save the life of the patient", combining the need for rapid intervention, thereby abolishing all possible interferences that, at this stage, could hinder the achievement of the target. Meanwhile, patients experience anxiety due to what is happening and, despite concerns about uncertainty over the postsurgical functional situation, delegate to the HCPs any decision. A similar phenomenon has already been described in the literature on the same population, defining it as resigned acceptance (Griffiths 
et al., 2008), because living is the ultimate aim (Reid et al., 2017).

Thus, the importance of "persuading" is crucial to the success of the whole process because HNC patients undergo a strong shock associated with their cancer diagnosis, consequent psycho-physical problems and uncertainty about his/her future (Lang et al., 2013). All the interferences described could be critical in these processes. For example, the cognitive tools or psychosocial needs of the patient could influence the choice because patients may opt for less radical but more conservative procedures from a morphological and functional point of view (Rana et al., 2016; Hahlweg et al., 2015; Hamilton et al., 2016).

Our research highlights how HCPs tend not to discuss the consequences of surgery (disfigurement and dysfunction) in the preoperative period to neutralize emerging interferences (Rana et al., 2016). This finding is consistent with those of Konradsen and colleagues (2009), who revealed how disfigurement was silenced in HCPs and HNC patient interactions to minimize this phenomenon. The process we described depicts the way in which a multidisciplinary team can support a decisionmaking process without the full involvement of the patient (Hahlweg et al., 2015; Hamilton et al., 2016) to rapidly eradicate a head and neck cancer.

Further interference could be represented by off-line HCPs, who might also consider less impacting procedures. Hamilton et al. (2016) revealed that when team members disagree about the treatment choice, the decision-making process could be unsuccessful. In the context studied, patient and family are involved in a well-defined preoperative pathway, that does not include professionals not strictly engaged in this preoperative phase. This is performed through a departmental culture in which management and communication models are well-established among the HCPs. The concept of a solid social relationship, as in a family, is believed by HCPs to be of vital importance to overcome the difficulties associated with surgery (Rana et al., 2016).

\section{Research limitations and future developments}

One of the limitations of this study is the lack of perspectives from patients who refused the surgery. 
Therefore, we do not know if the theory emerged from this study would have been confirmed by data collected from patients who refused the demolitive procedures. This type of participant was difficult to recruit because once they refused surgery, they shifted immediately to another department for conservative treatment. The only potential participant identified refused the interview.

Another limitation could be the absence of the family members' point of view. In fact, our theory shows that the family plays a "supporting role" in persuading and leading the patient to the surgery. This role emerges from narratives of patients and HCPs and was also confirmed by studies conducted in other contexts (Lang, et al, 2013; Rana et al., 2016; Richardson et al., 2015). This limitation can be explained by the difficulties experienced in involving family members in the short period of time that preceded the surgery.

Future studies could investigate the psycho-social process of HNC patients who refuse the surgery opting for other non-election therapeutic strategies. Deepening this issue could help HCPs to understand the motivations that can lead the patient to make different decisions than recommended. Moreover, further investigations could confirm the supportive role of the family, as we determined from the narratives of patients and HCPs.

\section{Clinical significance}

As a careful and individualized management of the preoperative phase could positively affect clinically relevant outcomes regarding the patient's quality of life (De Boer et al., 1998), the persuading process played by HCPs themselves could be reconsidered, including the appropriate content and communication approach that could be adopted with HNC patients.

The process studied is dominated by the need to move quickly to surgery, crippling possible fears and uncertainties related to the outcomes of the intervention. In this context, the study results could help HCPs understand the patient's experience in this preoperative phase. 


\section{REFERENCES}

Adelstein, D., Gillison, M. L., Pfister, D. G., Spencer, S., Adkins, D., Brizel, D. M., ... \& Colevas, A. D. (2017). NCCN guidelines insights: Head and neck cancers, version 2.2017. Journal of the National Comprehensive Cancer Network, 15(6), 761-770.

Bagnasco, A., Ghirotto, L., \& Sasso, L. (2015). La ricerca qualitativa: Una risorsa per i professionisti della salute. Edra Masson.

Charmaz K: Constructing grounded theory: A practical guide through qualitative analysis. 2006, London: Sage Publications

Chaturvedi, S. K., Shenoy, A., Prasad, K. M. R., Senthilnathan, S. M., \& Premlatha, B. S. (1996). Concerns, coping and quality of life in head and neck cancer patients. Supportive care in cancer, 4(3), 186-190.

De Boer, M. F.., Borne, B. V. D., Pruyn, J. F., Ryckman, R. M., Volovics, L., Knegt, P. P., ... \& Verwoerd, C. D. (1998). Psychosocial and physical correlates of survival and recurrence in patients with head and neck carcinoma: results of a 6-year longitudinal study. Cancer, 83(12), 2567-2579.

Dropkin, M. J. (2001). Anxiety, coping strategies, and coping behaviors in patients undergoing head and neck cancer surgery. Cancer Nursing, 24(2), 143-148.

Fife, B. L., \& Wright, E. R. (2000). The dimensionality of stigma: A comparison of its impact on the self of persons with HIV/AIDS and cancer. Journal of health and social behavior, 50-67.

Fingeret, M. C., Yuan, Y., Urbauer, D., Weston, J., Nipomnick, S., \& Weber, R. (2012). The nature and extent of body image concerns among surgically treated patients with head and neck cancer. Psycho $\square$ Oncology, 21(8), 836-844.

Finlay, L. (2002). "Outing” the Researcher: The Provenance, Process, and Practice of Reflexivity. Qualitative Health Research, 12(4), 531-545.

Glaser, B. G. (1978). Theoretical sensitivity: Advances in the methodology of 15 
grounded theory. Sociology Pr.

Griffiths, M. J., Humphris, G. M., Skirrow, P. M., \& Rogers, S. N. (2008). A qualitative evaluation of patient experiences when diagnosed with oral cancer recurrence. Cancer Nursing, 31(4), E11-E17.

Hahlweg, P., Hoffmann, J., Härter, M., Frosch, D. L., Elwyn, G., \& Scholl, I. (2015).

In absentia: an exploratory study of how patients are considered in multidisciplinary cancer team meetings. PLoS One, 10(10), e0139921.

Haisfield-Wolfe, M. E., McGuire, D. B., Soeken, K., Geiger-Brown, J., \& De Forge, B. R. (2009, May). Prevalence and correlates of depression among patients with head and neck cancer: a systematic review of implications for research. In Oncology nursing forum (Vol. 36, No. 3).

Hamilton, D. W., Heaven, B., Thomson, R. G., Wilson, J. A., \& Exley, C. (2016). Multidisciplinary team decision-making in cancer and the absent patient: a qualitative study. BMJ open, 6(7), e012559.

Harrison, L. B., Sessions, R. B., \& Hong, W. K. (Eds.). (2009). Head and neck cancer: a multidisciplinary approach. Lippincott Williams \& Wilkins.

Horney, D. J., Smith, H. E., McGurk, M., Weinman, J., Herold, J., Altman, K., \& Llewellyn, C. D. (2011). Associations between quality of life, coping styles, optimism, and anxiety and depression in pretreatment patients with head and neck cancer. Head \& neck, 33(1), 65-71.

Howren, M. B., Christensen, A. J., Hynds Karnell, L., Van Liew, J. R., \& Funk, G. F. (2013). Influence of pretreatment social support on health $\square$ related quality of life in head and neck cancer survivors: Results from a prospective study. Head \& neck, 35(6), 779-787.

Hutton, J. M., \& Williams, M. (2001). An investigation of psychological distress in patients who have been treated for head and neck cancer. British Journal of Oral and 
Maxillofacial Surgery, 39(5), 333-339.

Konradsen, H., Kirkevold, M., \& Zoffmann, V. (2009). Surgical facial cancer treatment: the silencing of disfigurement in nurse-patient interactions. Journal of advanced nursing, 65(11), 2409-2418.

Lang, H., France, E., Williams, B., Humphris, G., \& Wells, M. (2013). The psychological experience of living with head and neck cancer: a systematic review and meta $\square$ synthesis. Psycho $\square$ Oncology, 22(12), 2648-2663.

Lincoln, Y. S., \& Guba, E. G. (1985). Naturalistic inquiry. Newburry Park.

Llewellyn, C. D., McGurk, M., \& Weinman, J. (2005). Are psycho-social and behavioural factors related to health related-quality of life in patients with head and neck cancer? A systematic review. Oral Oncology, 41(5), 440-454.

Macgregor, F. C. (1990). Facial disfigurement: problems and management of social interaction and implications for mental health. Aesthetic plastic surgery, 14(1), 249257.

Mesters, I., Van den Borne, B., De Boer, M., \& Pruyn, J. (2001). Measuring information needs among cancer patients. Patient education and counseling, 43(3), 255-264.

Moore, K. A., Ford, P. J., \& Farah, C. S. (2014). Support needs and quality of life in oral cancer: a systematic review. International journal of dental hygiene, 12(1), 3647.

Ragaccio, A., Lombardo, A., \& Garrone, P. (2007). The quality of preoperative information to head and neck cancer patients: a qualitative study. Assistenza infermieristica e ricerca: AIR, 26(4), 200-209.

Rana, M., Czens, F., Wingartz, F., Gellrich, N. C., \& Rana, M. (2016). Doctors' tacit knowledge on coping processes of oral cancer patients: A qualitative study. Journal of Cranio-Maxillo-Facial Surgery, 44(12), 1977-1983. 
Reid, K. J., Swift, A., \& Mehanna, H. (2017). AQ methodology study to investigate the experiences of head and neck cancer patients from diagnosis to 1 year. European journal of cancer care, 26(6).

Richardson, A. E., Morton, R., \& Broadbent, E. (2015). Psychological support needs of patients with head and neck cancer and their caregivers: a qualitative study. Psychology \& health, 30(11), 1288-1305.

Scharloo, M., de Jong, B., Robert, J., Langeveld, T. P., van Velzen $\square$ Verkaik, E., Doorn $\square$ op den Akker, M. M., \& Kaptein, A. A. (2005). Quality of life and illness perceptions in patients with recently diagnosed head and neck cancer. Head \& Neck, 27(10), 857-863.

Threader, J., \& McCormack, L. (2016). Cancer $\square$ related trauma, stigma and growth: the 'lived'experience of head and neck cancer. European journal of cancer care, 25(1), 157-169.

Tong, A., Sainsbury, P., \& Craig, J. (2007). Consolidated criteria for reporting qualitative research (COREQ): a 32-item checklist for interviews and focus groups. International journal for quality in health care, 19(6), 349-357.

UK. CCR. UK Oral Cancer incidence statistics. 2011. Available at: http://www.cancerresearchuk.org/health-professional/cancer-statistics/statistics-bycancer-type/oral-cancer/incidence. Last access 04 April, 2018.

Van Der Molen, B. (1999). Relating information needs to the cancer experience: 1. Information as a key coping strategy. European Journal of Cancer Care, 8(4), 238. 


\section{Table 1 - Interview guide}

\begin{tabular}{|c|c|c|}
\hline & Persons indicated by the patient (PIP) & Healthcare professional and volunteers \\
\hline 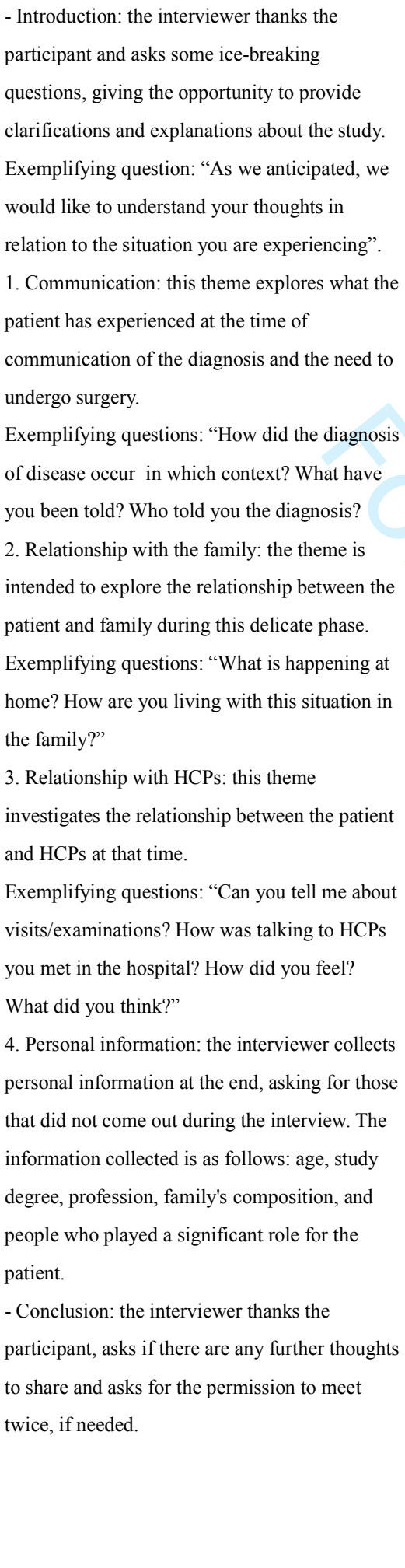 & 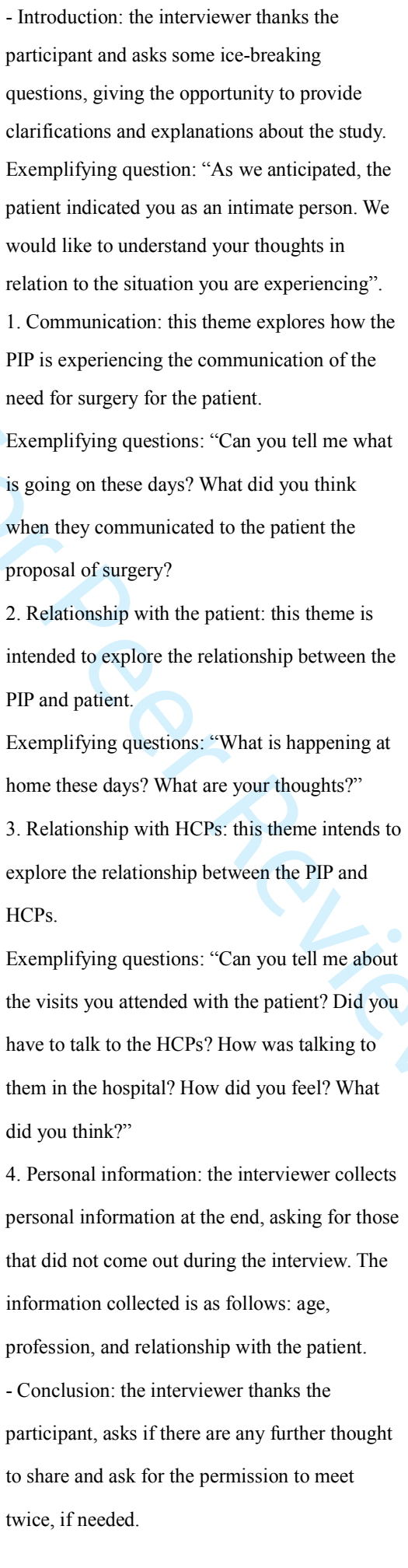 & 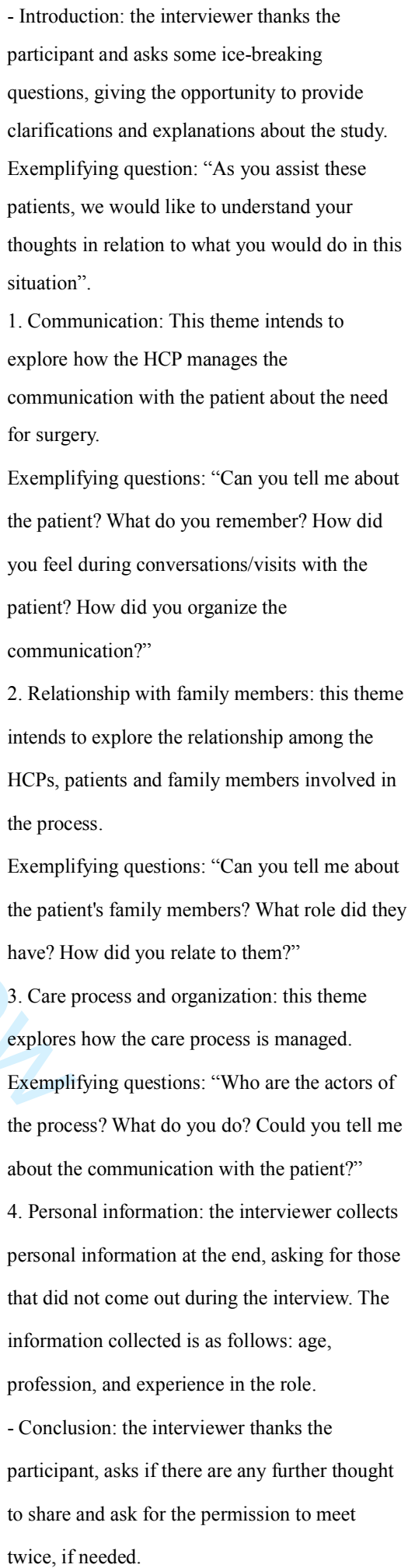 \\
\hline
\end{tabular}

$\mathrm{HCP}=$ healthcare professional; $\mathrm{PIP}=$ persons indicated by the patient. 
Table 2 - Final sample

\begin{tabular}{|c|c|c|c|c|c|c|c|c|c|}
\hline Participant & Sampling & $\begin{array}{l}\text { Co } \\
\text { de }\end{array}$ & Gender & $\begin{array}{c}\text { Ag } \\
\text { e }\end{array}$ & Profession & $\begin{array}{c}\text { Marital } \\
\text { status }\end{array}$ & Relatives & Interview setting & $\begin{array}{c}\text { Interview } \\
\text { length }\end{array}$ \\
\hline Patient & Initial & P1 & Male & 72 & Retired & Single & No & Patient room & $30^{\prime}$ \\
\hline Patient & Initial & $\mathrm{P} 2$ & Male & 71 & Employed & Married & Yes & Patient room & $40^{\prime}$ \\
\hline Patient & Initial & P3 & Male & 76 & Retired & Married & Yes & Patient room & $60^{\prime}$ \\
\hline Patient & Initial & P4 & Male & 54 & Employed & Partner & Yes & Nursing room & $25^{\prime}$ \\
\hline $\mathrm{HCP}$ & Initial & $\mathrm{HCP} 1$ & Male & 69 & Physician & & & Medical office & $30^{\prime}$ \\
\hline $\mathrm{HCP}$ & Initial & $\mathrm{HCP} 2$ & Female & 51 & Nurse & & & Nursing room & $30^{\prime}$ \\
\hline $\mathrm{HCP}$ & Initial & $\mathrm{HCP} 3$ & Female & 58 & Secretary & & & Medical office & $40^{\prime}$ \\
\hline $\mathrm{HCP}$ & Snowball & $\mathrm{HCP} 4$ & Female & 46 & Nurse & & & Medical office & $40^{\prime}$ \\
\hline Volunteer & Snowball & V1 & Male & 73 & Volunteer & & & Medical office & $90^{\prime}$ \\
\hline Patient & Theoretical & P5 & Male & 80 & Retired & Married & Yes & Patient room & $60^{\prime}$ \\
\hline Patient & Theoretical & P6 & Female & 66 & Retired & Married & Yes & Patient room & $40^{\prime} \& 20^{\prime}$ \\
\hline $\mathrm{HCP}$ & Theoretical & HCP67 & Female & 49 & $\begin{array}{l}\text { Speech } \\
\text { therapist }\end{array}$ & & & Medical office & $25^{\prime}$ \\
\hline Volunteer & Theoretical & $\mathrm{V} 2$ & Male & 72 & Volunteer & & & Association & $65^{\prime}$ \\
\hline $\mathrm{HCP}$ & Theoretical & НСР79 & Female & 38 & $\begin{array}{l}\text { Speech } \\
\text { therapist }\end{array}$ & & & Medical office & $40^{\prime}$ \\
\hline $\mathrm{HCP}$ & Theoretical & НCP810 & Female & 52 & $\begin{array}{l}\text { Speech } \\
\text { therapist }\end{array}$ & & & Medical office & $45^{\prime}$ \\
\hline $\mathrm{HCP}$ & Theoretical & НСР911 & Male & 58 & Physician & & & Medical office & $35^{\prime}$ \\
\hline
\end{tabular}


Figure 1 - Theoretical model

No legends 


\title{
TITLE
}

\section{Undergoing Head and Neck cancer surgery: a Grounded Theory}

\begin{abstract}
Objective: Surgery is the treatment of choice in most head and neck cancers. Very often, the surgery is demolitive with high impact on the psycho-social, functional and aesthetic fields.

Methods: We conducted a grounded theory study with semi-structured interviews to explore the psycho-social process occurring when a patient with head and neck cancer needs to undergo surgery.

Results: Seventeen participants (six patients, nine health care professionals, and two volunteers) were interviewed immediately before surgery. The study generated a process of "persuading the patient of an obligation" as the core category. The other principal categories that emerged highlighted the patients' doubts and fears regarding the surgery consequences and, in parallel, strategies employed by the health care professionals to contrast hindering issues impeding surgery. In particular, healthcare professionals involved patients in an affiliation process through simplified communication to sustain the choice of surgery; the family plays a supportive role in this process.

Conclusion: The interplay between the organizational process and patients' experience results in "I will let you convince me" at the end of the decision making process, where the main aim was to save and be saved.
\end{abstract}

Keywords: Head and neck cancer, Grounded theory, Surgery, Decision Making, Patient participation, Preoperative care 
Head and neck cancer (HNC) refers to a group of tumours that develop in the body region of the head and neck and may affect the oral cavity, pharynx, larynx, nasal cavities, paranasal sinuses, and salivary glands. The incidence is estimated to be above half a million cases per year worldwide and represents, by diffusion, the fifth most common type of cancer (Harrison et al., 2009). The main factors that influence its development are alcohol and tobacco abuse (UK CCR, 2011). Due to the development of diagnostic-therapeutic techniques, the overall survival rate for this group of malignancies has slightly improved over the past decade, particularly for individuals with the highest socio-economic level (UK CCR, 2011). Most HNC cases are surgically treated, and their outcomes are often demolitive (Adelstein et al., 2017). Similar to other oncological diseases, the diagnosis of an HNC has a significant psychosocial impact on patients (Chaturvedi et al., 1996) who may experience uncertainty about the consequences of surgery on their physical, functional and psychological well-being (Hutton \& Williams, 2001). The facial region is an important aspect of personal identity, and changes in its image often cause intense suffering and embarrassment (Macgregor, 1990; Fingeret et al., 2012). Moreover, many patients have to face significant changes in functions such as communication, breathing, swallowing and the sense of taste (Harrison et al., 2009; Howren et al., 2013). In particular, the disfigurement and impairment that often result from surgical treatment may emerge as stigmatization (Macgregor, 1990) because patients may blame themselves for their risky behaviours (e.g., smoking and alcohol abuse) (Fife \& Wright, 2000).

From $15 \%$ to $50 \%$ of patients report clinically relevant anxiety and depression (Haisfield-Wolfe et al., 2009; Llewellyn et al., 2005; Threader \& McCormack, 2016). Most studies on the psychosocial aspects of $\mathrm{HNC}$ are quantitative. They have highlighted the prevalence of anxiety and depression in this population and the relationship between these symptoms and coping skills, quality of life and other variables related to treatment outcomes (Dropkin, 2001; Horney et al., 2011; Moore et al., 2014). Only recently have these aspects also been studied using qualitative approaches. These studies primarily investigate the consequences of surgical treatment (Lang et al., 2013), while the 
understanding of how patients experience the pre-surgery phase is underdeveloped. The few qualitative contributions that discuss the pre-surgical phase mostly address the topic of information/communication related to treatment and possible consequences (Ragaccio et al., 2007; Sharloo et al., 2005). Information received in the preoperative phase was often provided too quickly and was perceived as too detailed and too complicated for the emotional status of the patients, thus affecting their decision-making process (Ragaccio et al., 2007; Sharloo et al., 2005). This incomplete awareness could adversely affect the patient's coping strategies and prognosis (Sharloo et al., 2005), as also reported in different oncological populations (Mesters et al., 2001; Van Der Molen, 1999).

A grounded theory (GT) study (Konrades et al., 2009) revealed how profoundly impacting issues, such as disfigurement, are not addressed appropriately in the literature. Dropkin (2001) showed that when health professionals anticipate the disfigurement issues before surgery, patients would respond by developing high levels of anxiety and lower coping abilities. On the other hand, a high level of uncertainty about surgical consequences would adversely affect the prognosis (De Boer et al., 1998).

Based on current knowledge, no study has focused on the HNC patients' perspectives in the presurgical phase.

Understanding the patients' experience from the diagnosis to the surgical treatment would be beneficial to improve the quality of pathways considering their practical, psychological, and relational needs. This study was aimed to explore the psycho-social process that occurs when a patient with $\mathrm{HNC}$ cancer receives communication about the need to undergo surgery. Our research question is as follows: "what is going on when healthcare professionals propose surgery to an HNC patient?" 


\section{METHODS}

\section{Methodological framework}

To address the research question, we followed the Charmaz's constructivist grounded theory approach (GT) (2006). GT is a general method of interpretative research adopted by social scientists to define the processes underlying interpersonal interactions (Charmaz, 2006; Glaser, 1978). We adopted GT to develop a theoretical model that explains which factors may influence the interpersonal process and which challenges patients, their families and the HCP are facing.

\section{Setting and sampling}

The GT study was carried out at the otolaryngology ward of the Reggio Emilia Hospital "Arcispedale Santa Maria Nuova” (Azienda Sanitaria Locale-IRCCS). The researchers identified two additional contexts for the research: the Azienda Ospedaliero-Universitaria Policlinico of Modena and the Hospital of Sassuolo (Modena).

\section{Inclusion criteria}

The HNC patient candidate for surgery and his/her family, caregivers and HCP represented the unit of analysis of this study.

Our sample included the following:

- adults diagnosed with HNC, waiting for surgical treatment

- family members and/or caregivers considered significant by the patient

- subjects who comprehend and speak Italian

- HCPs who work in clinical settings involved in this research

\section{Sampling procedures}

The sampling followed three steps: initial sampling, snowball sampling, and theoretical sampling 
(Charmaz, 2006; Bagnasco, 2015). The initial sampling was purposive (programmed in advance) based on the research question. It involved four HNC patients, candidates for demolitive surgery, and three HCPs.

Thereafter, we applied snowball sampling where individuals already recruited indicated further potential participants (caregivers or HCPs) who played a significant role for them. At the end of this step, we included two more people, an HCP and a volunteer. During the data analysis, we applied the theoretical sampling to confirm and saturate the provisional categories involving two HNC patients, one volunteer and six additional HCPs.

\section{Data collection}

The E.L. (MScSpT, speech therapist), M.G. (MScN, nurse manager), M.C.B. (MSc, librarian and information specialist), and S.C. (MScPT, physiotherapist) collected data between May 2016 and January 2017. All researchers were female, had no professional relationship with the patients and did not work in the wards where the data were collected. They received one year of training in qualitative research methodology. When possible, the interviewer was introduced face-to face with the patient by the head nurse. All the interviews were performed in an appropriate place in the clinic (workplace for HCP) by an interviewer and an observer. Prior to the beginning of the interview, researchers explained to the participants the aim of the study that is, the need to deeply understand the process triggered when a patient is told the necessity to undergo surgery to remove a cancer in the head and neck region. Three semi-structured open-ended interviews were prepared: one for the HNC patients, one for persons indicated by the patients (PIP), and one for the HCPs (Table 1). Questions were asked in different orders, and themes were treated according to the priorities of the respondents. The interviewers explored all the key themes, posing clarification questions and exemplification requests. Additionally, the interviewer asked the participants about the opportunity to meet a second time in the case of doubts in the interpretation of the collected data. The researchers collected demographic information, qualitative data and field notes concerning nonverbal behaviours of the participants. 
The interviews lasted between 20 and 60 minutes. The researchers audio recorded and transcribed verbatim the interviews within 48 hours from their completion. The participants were given the opportunity to read the transcript if requested. One patient asked to read the interview and confirmed the content.

\section{Rigor}

Two researchers collected the data on the field, whether the other two researchers played a role in the audit, checking the interview transcripts and coding. The four researchers who collected the data also open-coded the interview transcription line by line using the participants' words. The credibility of the explanatory model was pursued by collecting both in-depth data and field notes.

Moreover, the authors critically discussed and verified the relevance of the levels of coding, coherence of categorization made during focused coding and data saturation (Lincoln \& Guba, 1995; Finley, 2002).

\section{Data analysis and saturation}

The researchers started data analysis concurrently with data collection, following the GT indications for coding (Charmaz, 2006):

1. Open coding: researchers who conducted the interviews indexed them using codes and then shared the data with colleagues.

2. Focused coding: researchers grouped the codes into conceptual categories, identifying concepts at a higher level of abstraction. The first conceptualization was derived from the data of the first seven interviews from which the researchers identified nine conceptual categories.

3. Theoretical coding: researchers defined the explanatory theoretical model, highlighting the relationships between the conceptual categories. This phase has allowed for the data to be summarized in one core category plus three principal categories and their related subcategories. Researchers performed the theoretical coding in group under the supervision 
of L.G. and S.D.L. as external auditors. They stopped recruiting participants when the analysis reached data saturation: the latest interviews revealed no new information or insights.

\section{Ethical considerations}

The Provincial Ethics Committee of Reggio Emilia approved the study (Protocol n. 2013/0009390 of 2013/04/09). The research was conducted following ICH E6 Guidelines for the GCP and the principles of the Declaration of Helsinki.

The study and its report followed the consolidated criteria for explicit and comprehensive reporting of qualitative research checklist (Tong et al., 2007)

\section{RESULTS}

The final sample included 17 participants (six patients, nine HCPs and two volunteers) whose characteristics are reported in Table 2. Researchers conducted 18 interviews: P6 was interviewed twice to clarify some doubts and gather more information. During the interviews, only patients and researchers were present. In one case, the patient's wife was present for half of the interview duration (P3). HNC patients did not indicate any PIP.

\section{Explanatory theoretical model}

Based on the conceptual categories emerging from the data, we developed an interpretative model to answer the research question, "What is going on when healthcare professionals propose surgery to an HNC patient?". The theoretical model develops through three phases and explains the psychosocial process that occurs when a patient with $\mathrm{HNC}$ is receiving communication about the need to undergo surgery (Figure 1).

\section{Core category: persuading the patient of an obligation}


What happens in the context studied is a process where HCPs employ strategies to convince the patient to accept the surgery, and conversely, the patients allow themselves to be convinced. What we call "persuading the patient of an obligation" is the core category, which connects all the categories into a conceptual framework. The term "persuading" is used in the sense of "influence to act, to make a decision". In our data, the pivotal concept is surgery seen as an obligation.

"I told him [to the patient] that the most appropriate therapy for the disease according to international guidelines is the surgery... You have to do" (HCP1).

"I told her: you have a malignant laryngeal cancer, and we have two options: one is radiotherapy, and the other is surgery. We decided to do surgery" (HCP5).

"Because... there are no alternatives... there is nothing to do. So, I calmed my heart down, and I accept what comes" (P4).

\section{Description of principal category: interferences}

The process of "persuading the patient of an obligation" begins with a first phase in which some impeding factors have been identified as "interferences". Those factors are manifold and concern the following: 1) shock for the diagnosis experienced by the patients; 2) patients' expectations regarding the functional outcomes of the surgery; 3) cognitive and educational levels of the patients; and 4) professionals who potentially might be "offline".

1) Patients receive a diagnosis of cancer and almost immediately the proposal for surgical treatment. Patients are very scared.

"It is going to be bad... what do you want me to say? [palms to the ceiling] I would throw myself out of the window" (P5).

"I am afraid I cannot... I have a throat cancer... and I am desperate..." (P1).

2) The expectations regarding the functional outcomes of the surgery may delay the acceptance of the surgical procedures: the patients' attention seems to be focused on the life-changing adaptations, regarding the voice, speech, and swallowing changes, rather than on the cancer. 
"Well... my fear is to totally change my life, in the sense that... for what my doctor anticipated... I will have to change both the way I eat and the way I talk... so... a fear... remarkable that I have because I do not know when I will wake up... under what conditions" (P3).

"They [the patients] mystified and try to assume an attitude as if it was not the disease to be solved but the voice problem, so they ask if there will be a way to talk and feed... without facing the 'cancer' topic" (HCP1).

"...they [the changes] are difficult to accept, are life-changing. They change the lives of family members, in many ways, not only from the point of view of the voice, speech, eating and swallowing, but in the very intimate sphere of relationships with the family, with the wife, husband..." (HCP6).

3) The cognitive and educational levels of patients may influence the acceptance of surgery. "When patients have a certain type of high or medium-high schooling, or have an activity where voice is crucial... here, it becomes difficult because the voice becomes dominant... they see their world collapsing... and then other difficulties when they are informed from the internet. They believe they know everything, and then... say what they want you to do without having the awareness of the problem... For less educated people, the word 'cancer' is still like a divine curse, so they have the concept of saving the skin. I am the one who saves their skin" (HCP1).

4) The presence of professionals who might be "offline" can interfere with the need to persuade patients of the obligation. The "offline" professionals are the newly hired with little familiarity with HNC patients or those considered as outside the "culture" of the otolaryngology ward.

"The surgical decision does not involve us or at least not in this hospital, not in this type of management and... I have never been summoned to the tumour board" (HCP6).

"What helps us is the fact that we are all tenure nurses, and we have worked together for many years... we all have the same way of managing and experiencing these situations... 
The new staff does not deal with these types of patients. A model has been created... we all do the same thing... those who came after... at the beginning... have a different way of managing... but after a while, they do as we do" (HCP2).

\section{Description of principal category: contrasting hindering issues}

In this phase, the interferences to surgery adherence are managed by a twofold strategy, which includes affiliation and neutralization attempts. Furthermore, the family may play an important role in contrasting issues that could delay surgery.

Regarding the affiliation attempt, data analysis showed that HCPs act within a departmental culture and build a unified and shared communication modality. HCPs are very confident about themselves and their way of working.

"When we have a patient to whom we have to do some kind of intervention or tell him about a diagnosis, we always do it collegially. Let us first talk about it and decide together what to say, and then... we share the same attitude and keep the same version" (HCP1).

"Beyond standardized procedures, we have adopted a behaviour... this happens daily because these are the patients we see for a long time. They are so specific that they can only be managed here, with our features" (HCP2).

The HCPs are committed to build a departmental culture in which we feel like a family and tend to define patients as "our" patients:

"When a family relationship is established, we are their family" (HCP4).

Furthermore, these strategies contribute to gain total trust from the patients.

"I trust the professor. I sought information from other doctors, but they earned my trust here. The doctor said he will do all he can. They [the HCPs] have studied a lot! I gave him a white paper. Even if he does something that is not good, if it is necessary, he needs to do it. Even if there are two or three ways, I told him 'you choose the best'. If it is possible, I do not want the hole. However, they do what they can" (P2). 
"I trust here, and that means so much" (P3).

The neutralization attempt explains how patients and HCPs justify surgery and adhere to it. HCPs and patients seem to agree in the stereotypical explanation of the pathology, while HCPs stigmatize the idea of HNC patients and patients blame themselves for the disease.

"Our patients are, in the vast majority, marginalized and... on the margins of society, in the sense that they are people who drink and smoke... people who have a disorderly life, especially in terms of smoking and alcohol consumption" (HCP1).

"It is my fault, I was smoking... it depends on smoke... if I tell you everything I did...Therefore, I was looking for it" (P1).

"If I had stopped drinking and smoking... I had been following the line, probably it [the cancer] would not jump out. Now I am paying for it. You are the one who wanted it. This is the truth" (P2).

The communication is simplified to reassure patients and provide them with general information. HCPs minimize potential side effects of surgery to neutralize fear and shock from the patients. They give them no specific information about foreseeable future conditions and delay all possible explanations to the postoperative phase.

"They are very frightened at first. What will happen is not clear to them because they have not understood what it means. In the days to follow [after the surgery], as soon as the situation is calmed down, when they realize that life continues, we will accompany them. We will explain things as if we were explaining to a child" (HCP2).

Sometimes, the possible consequences of surgery are minimized:

"I say to the relatives... 'You do not have to feel sorry for him. This is still good [touching the head], we miss the voice, but we have the head. You have to live the life you had before... going to the café, reading the newspaper, eating fruits, the meat" (V1).

The role of the family is important in persuading the patients to undergo surgery, supporting the 
team decisions and taking care of the emotional needs of their beloved.

"We try to figure out who the person is [the patient]... if we have to first approach the family to get to him" (HCP1).

"The situation depends very much on the family climate. If there are patients followed by relatives, giving support, it would be easier. All that can be a support, an incitement, an exhortation from the family helps" (HCP6).

"My family, honestly, for me... they support me, and they tell me 'you will see... everything will be put in place' " (P3).

\section{Description of principal category: "I will let you convince me"}

The process we studied ends with the patient's adherence to the proposal. If the affiliation and neutralization attempts work, the patients allow themselves to be persuaded to undergo surgery, representing "salvation" for all the participants.

"I have to do it. It is in my mind. If you want to live, you have to do it" (P4).

"And now we have to do this. I decided... to do... to live as I will can" (P3).

\section{DISCUSSION}

All the main categories emerged, converge on persuading the patient that surgery is a "salvation", and as a result, persuading the patient to undergo surgery is the core category. The process of persuasion and being persuaded develops within a patient-HCP relationship dominated by the need to have surgery to save and be saved.

The conceptual model developed explains the interplay between the organizational process and patients' experience. In fact, the HCPs activate the therapeutic pathway necessary to "save the life of the patient", combining the need for rapid intervention, thereby abolishing all possible interferences that, at this stage, could hinder the achievement of the target. Meanwhile, patients 
experience anxiety due to what is happening and, despite concerns about uncertainty over the postsurgical functional situation, delegate to the HCPs any decision. A similar phenomenon has already been described in the literature on the same population, defining it as resigned acceptance (Griffiths et al., 2008), because living is the ultimate aim (Reid et al., 2017).

Thus, the importance of "persuading" is crucial to the success of the whole process because HNC patients undergo a strong shock associated with their cancer diagnosis, consequent psycho-physical problems and uncertainty about his/her future (Lang et al., 2013). All the interferences described could be critical in these processes. For example, the cognitive tools or psychosocial needs of the patient could influence the choice because patients may opt for less radical but more conservative procedures from a morphological and functional point of view (Rana et al., 2016; Hahlweg et al., 2015; Hamilton et al., 2016).

Our research highlights how HCPs tend not to discuss the consequences of surgery (disfigurement and dysfunction) in the preoperative period to neutralize emerging interferences (Rana et al., 2016). This finding is consistent with those of Konradsen and colleagues (2009), who revealed how disfigurement was silenced in HCPs and HNC patient interactions to minimize this phenomenon. The process we described depicts the way in which a multidisciplinary team can support a decisionmaking process without the full involvement of the patient (Hahlweg et al., 2015; Hamilton et al., 2016) to rapidly eradicate a head and neck cancer.

Further interference could be represented by off-line HCPs, who might also consider less impacting procedures. Hamilton et al. (2016) revealed that when team members disagree about the treatment choice, the decision-making process could be unsuccessful. In the context studied, patient and family are involved in a well-defined preoperative pathway, that does not include professionals not strictly engaged in this preoperative phase. This is performed through a departmental culture in which management and communication models are well-established among the HCPs. The concept of a solid social relationship, as in a family, is believed by HCPs to be of vital importance to overcome the difficulties associated with surgery (Rana et al., 2016). 


\section{Research limitations and future developments}

One of the limitations of this study is the lack of perspectives from patients who refused the surgery. Therefore, we do not know if the theory emerged from this study would have been confirmed by data collected from patients who refused the demolitive procedures. This type of participant was difficult to recruit because once they refused surgery, they shifted immediately to another department for conservative treatment. The only potential participant identified refused the interview.

Another limitation could be the absence of the family members' point of view. In fact, our theory shows that the family plays a "supporting role" in persuading and leading the patient to the surgery. This role emerges from narratives of patients and HCPs and was also confirmed by studies conducted in other contexts (Lang, et al, 2013; Rana et al., 2016; Richardson et al., 2015). This limitation can be explained by the difficulties experienced in involving family members in the short period of time that preceded the surgery.

Future studies could investigate the psycho-social process of HNC patients who refuse the surgery opting for other non-election therapeutic strategies. Deepening this issue could help HCPs to understand the motivations that can lead the patient to make different decisions than recommended. Moreover, further investigations could confirm the supportive role of the family, as we determined from the narratives of patients and HCPs.

\section{Clinical significance}

As a careful and individualized management of the preoperative phase could positively affect clinically relevant outcomes regarding the patient's quality of life (De Boer et al., 1998), the persuading process played by HCPs themselves could be reconsidered, including the appropriate content and communication approach that could be adopted with HNC patients.

The process studied is dominated by the need to move quickly to surgery, crippling possible fears 
and uncertainties related to the outcomes of the intervention. In this context, the study results could help HCPs understand the patient's experience in this preoperative phase. 


\section{REFERENCES}

Adelstein, D., Gillison, M. L., Pfister, D. G., Spencer, S., Adkins, D., Brizel, D. M., ... \& Colevas, A. D. (2017). NCCN guidelines insights: Head and neck cancers, version 2.2017. Journal of the National Comprehensive Cancer Network, 15(6), 761-770.

Bagnasco, A., Ghirotto, L., \& Sasso, L. (2015). La ricerca qualitativa: Una risorsa per i professionisti della salute. Edra Masson.

Charmaz K: Constructing grounded theory: A practical guide through qualitative analysis. 2006, London: Sage Publications

Chaturvedi, S. K., Shenoy, A., Prasad, K. M. R., Senthilnathan, S. M., \& Premlatha, B. S. (1996). Concerns, coping and quality of life in head and neck cancer patients. Supportive care in cancer, 4(3), 186-190.

De Boer, M. F.., Borne, B. V. D., Pruyn, J. F., Ryckman, R. M., Volovics, L., Knegt, P. P., ... \& Verwoerd, C. D. (1998). Psychosocial and physical correlates of survival and recurrence in patients with head and neck carcinoma: results of a 6-year longitudinal study. Cancer, 83(12), 2567-2579.

Dropkin, M. J. (2001). Anxiety, coping strategies, and coping behaviors in patients undergoing head and neck cancer surgery. Cancer Nursing, 24(2), 143-148.

Fife, B. L., \& Wright, E. R. (2000). The dimensionality of stigma: A comparison of its impact on the self of persons with HIV/AIDS and cancer. Journal of health and social behavior, 50-67.

Fingeret, M. C., Yuan, Y., Urbauer, D., Weston, J., Nipomnick, S., \& Weber, R. (2012). The nature and extent of body image concerns among surgically treated patients with head and neck cancer. Psycho $\square$ Oncology, 21(8), 836-844.

Finlay, L. (2002). "Outing” the Researcher: The Provenance, Process, and Practice of Reflexivity. Qualitative Health Research, 12(4), 531-545.

Glaser, B. G. (1978). Theoretical sensitivity: Advances in the methodology of 16 
grounded theory. Sociology Pr.

Griffiths, M. J., Humphris, G. M., Skirrow, P. M., \& Rogers, S. N. (2008). A qualitative evaluation of patient experiences when diagnosed with oral cancer recurrence. Cancer Nursing, 31(4), E11-E17.

Hahlweg, P., Hoffmann, J., Härter, M., Frosch, D. L., Elwyn, G., \& Scholl, I. (2015).

In absentia: an exploratory study of how patients are considered in multidisciplinary cancer team meetings. PLoS One, 10(10), e0139921.

Haisfield-Wolfe, M. E., McGuire, D. B., Soeken, K., Geiger-Brown, J., \& De Forge, B. R. (2009, May). Prevalence and correlates of depression among patients with head and neck cancer: a systematic review of implications for research. In Oncology nursing forum (Vol. 36, No. 3).

Hamilton, D. W., Heaven, B., Thomson, R. G., Wilson, J. A., \& Exley, C. (2016). Multidisciplinary team decision-making in cancer and the absent patient: a qualitative study. BMJ open, 6(7), e012559.

Harrison, L. B., Sessions, R. B., \& Hong, W. K. (Eds.). (2009). Head and neck cancer: a multidisciplinary approach. Lippincott Williams \& Wilkins.

Horney, D. J., Smith, H. E., McGurk, M., Weinman, J., Herold, J., Altman, K., \& Llewellyn, C. D. (2011). Associations between quality of life, coping styles, optimism, and anxiety and depression in pretreatment patients with head and neck cancer. Head \& neck, 33(1), 65-71.

Howren, M. B., Christensen, A. J., Hynds Karnell, L., Van Liew, J. R., \& Funk, G. F. (2013). Influence of pretreatment social support on health $\square$ related quality of life in head and neck cancer survivors: Results from a prospective study. Head \& neck, 35(6), 779-787.

Hutton, J. M., \& Williams, M. (2001). An investigation of psychological distress in patients who have been treated for head and neck cancer. British Journal of Oral and 
Maxillofacial Surgery, 39(5), 333-339.

Konradsen, H., Kirkevold, M., \& Zoffmann, V. (2009). Surgical facial cancer treatment: the silencing of disfigurement in nurse-patient interactions. Journal of advanced nursing, 65(11), 2409-2418.

Lang, H., France, E., Williams, B., Humphris, G., \& Wells, M. (2013). The psychological experience of living with head and neck cancer: a systematic review and meta $\square$ synthesis. Psycho $\square$ Oncology, 22(12), 2648-2663.

Lincoln, Y. S., \& Guba, E. G. (1985). Naturalistic inquiry. Newburry Park.

Llewellyn, C. D., McGurk, M., \& Weinman, J. (2005). Are psycho-social and behavioural factors related to health related-quality of life in patients with head and neck cancer? A systematic review. Oral Oncology, 41(5), 440-454.

Macgregor, F. C. (1990). Facial disfigurement: problems and management of social interaction and implications for mental health. Aesthetic plastic surgery, 14(1), 249257.

Mesters, I., Van den Borne, B., De Boer, M., \& Pruyn, J. (2001). Measuring information needs among cancer patients. Patient education and counseling, 43(3), 255-264.

Moore, K. A., Ford, P. J., \& Farah, C. S. (2014). Support needs and quality of life in oral cancer: a systematic review. International journal of dental hygiene, 12(1), 3647.

Ragaccio, A., Lombardo, A., \& Garrone, P. (2007). The quality of preoperative information to head and neck cancer patients: a qualitative study. Assistenza infermieristica e ricerca: AIR, 26(4), 200-209.

Rana, M., Czens, F., Wingartz, F., Gellrich, N. C., \& Rana, M. (2016). Doctors' tacit knowledge on coping processes of oral cancer patients: A qualitative study. Journal of Cranio-Maxillo-Facial Surgery, 44(12), 1977-1983. 
Reid, K. J., Swift, A., \& Mehanna, H. (2017). AQ methodology study to investigate the experiences of head and neck cancer patients from diagnosis to 1 year. European journal of cancer care, 26(6).

Richardson, A. E., Morton, R., \& Broadbent, E. (2015). Psychological support needs of patients with head and neck cancer and their caregivers: a qualitative study. Psychology \& health, 30(11), 1288-1305.

Scharloo, M., de Jong, B., Robert, J., Langeveld, T. P., van Velzen $\square$ Verkaik, E., Doorn $\square$ op den Akker, M. M., \& Kaptein, A. A. (2005). Quality of life and illness perceptions in patients with recently diagnosed head and neck cancer. Head \& Neck, 27(10), 857-863.

Threader, J., \& McCormack, L. (2016). Cancer $\square$ related trauma, stigma and growth: the 'lived'experience of head and neck cancer. European journal of cancer care, 25(1), 157-169.

Tong, A., Sainsbury, P., \& Craig, J. (2007). Consolidated criteria for reporting qualitative research (COREQ): a 32-item checklist for interviews and focus groups. International journal for quality in health care, 19(6), 349-357.

UK. CCR. UK Oral Cancer incidence statistics. 2011. Available at: http://www.cancerresearchuk.org/health-professional/cancer-statistics/statistics-bycancer-type/oral-cancer/incidence. Last access 04 April, 2018.

Van Der Molen, B. (1999). Relating information needs to the cancer experience: 1. Information as a key coping strategy. European Journal of Cancer Care, 8(4), 238. 


\section{Table 1 - Interview guide}

\begin{tabular}{|c|c|c|}
\hline & ersons indicated by the patient (PIP) & Healthcare professional and volunteers \\
\hline 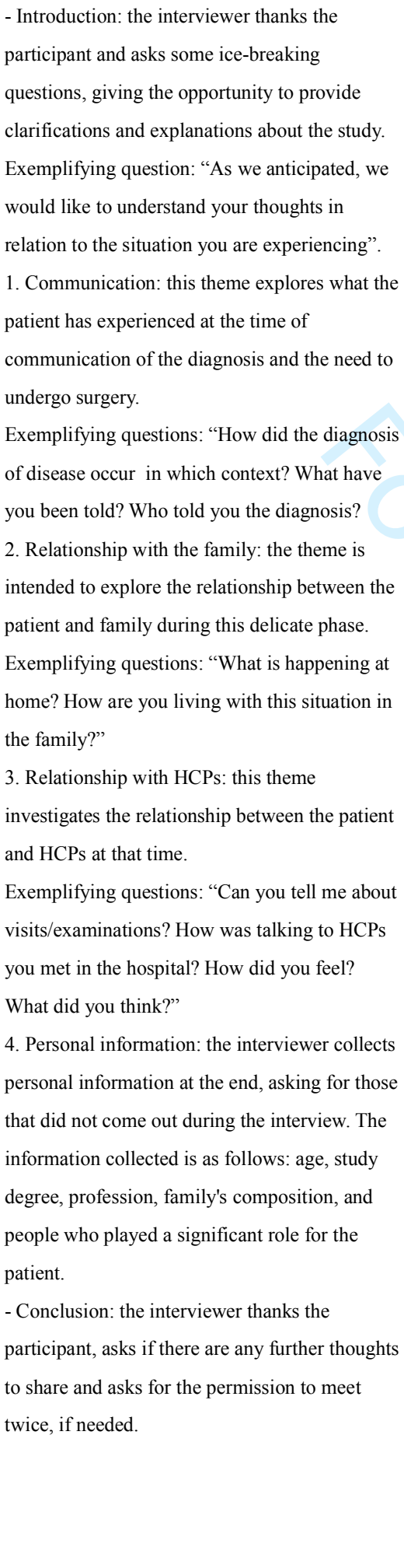 & 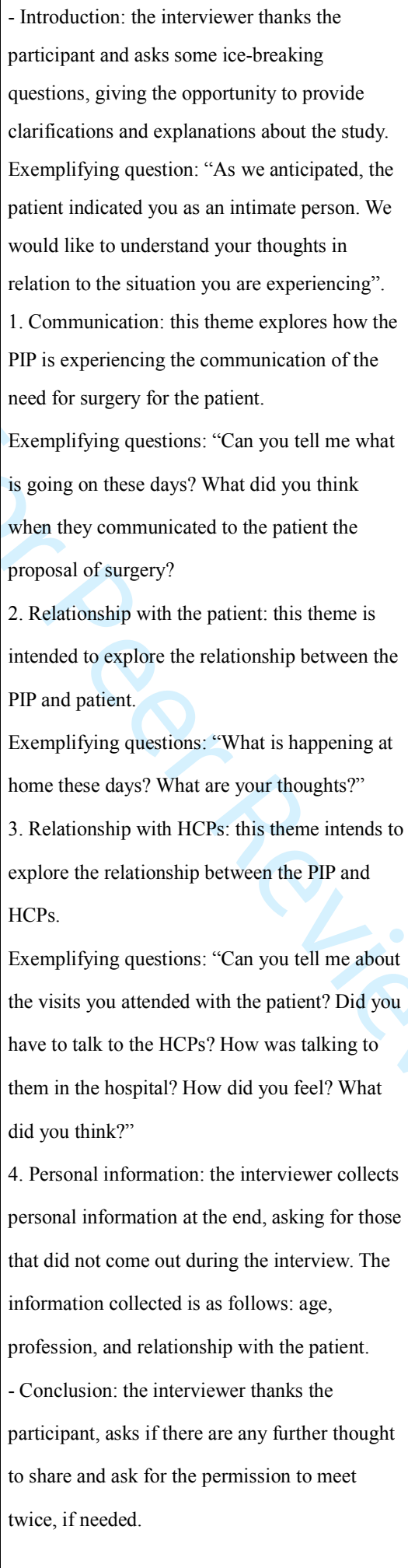 & 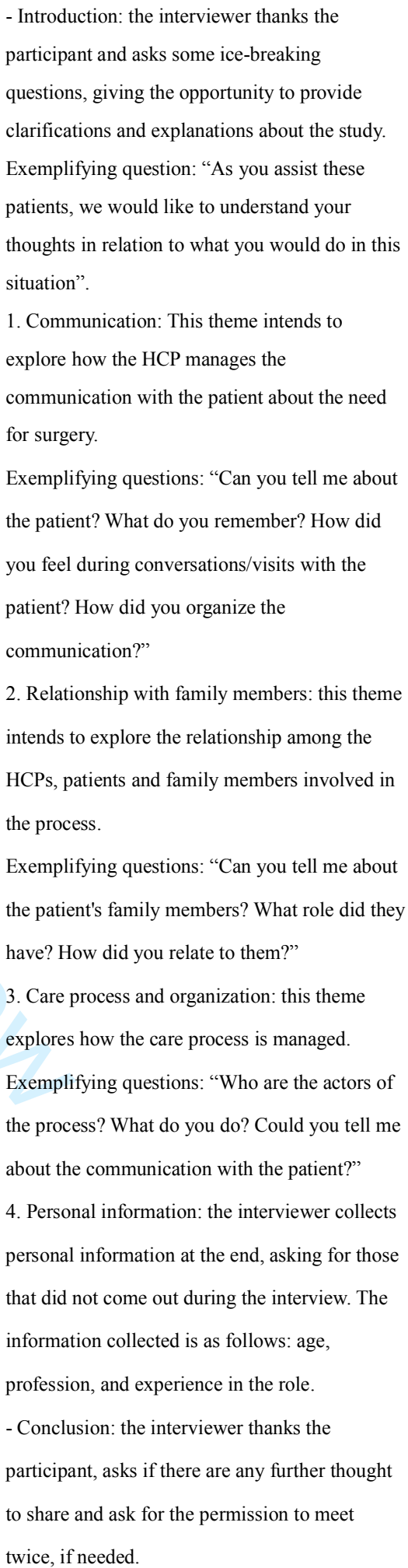 \\
\hline
\end{tabular}

$\mathrm{HCP}=$ healthcare professional; $\mathrm{PIP}=$ persons indicated by the patient. 
Table 2 - Final sample

\begin{tabular}{|c|c|c|c|c|c|c|c|c|c|}
\hline Participant & Sampling & $\begin{array}{l}\text { Co } \\
\text { de }\end{array}$ & Gender & $\begin{array}{c}\text { Ag } \\
\text { e }\end{array}$ & Profession & $\begin{array}{c}\text { Marital } \\
\text { status }\end{array}$ & Relatives & Interview setting & $\begin{array}{c}\text { Interview } \\
\text { length }\end{array}$ \\
\hline Patient & Initial & $\mathrm{P} 1$ & Male & 72 & Retired & Single & No & Patient room & $30^{\prime}$ \\
\hline Patient & Initial & $\mathrm{P} 2$ & Male & 71 & Employed & Married & Yes & Patient room & $40^{\prime}$ \\
\hline Patient & Initial & P3 & Male & 76 & Retired & Married & Yes & Patient room & $60^{\prime}$ \\
\hline Patient & Initial & P4 & Male & 54 & Employed & Partner & Yes & Nursing room & $25^{\prime}$ \\
\hline $\mathrm{HCP}$ & Initial & HCP1 & Male & 69 & Physician & & & Medical office & $30^{\prime}$ \\
\hline $\mathrm{HCP}$ & Initial & $\mathrm{HCP} 2$ & Female & 51 & Nurse & & & Nursing room & $30^{\prime}$ \\
\hline $\mathrm{HCP}$ & Initial & $\mathrm{HCP} 3$ & Female & 58 & Secretary & & & Medical office & $40^{\prime}$ \\
\hline $\mathrm{HCP}$ & Snowball & $\mathrm{HCP} 4$ & Female & 46 & Nurse & & & Medical office & $40^{\prime}$ \\
\hline Volunteer & Snowball & V1 & Male & 73 & Volunteer & & & Medical office & $90^{\prime}$ \\
\hline Patient & Theoretical & P5 & Male & 80 & Retired & Married & Yes & Patient room & $60^{\prime}$ \\
\hline Patient & Theoretical & P6 & Female & 66 & Retired & Married & Yes & Patient room & $40^{\prime} \& 20^{\prime}$ \\
\hline $\mathrm{HCP}$ & Theoretical & НCР56 & Female & 62 & Physician & & & Medical office & $20^{\prime}$ \\
\hline $\mathrm{HCP}$ & Theoretical & НСР67 & Female & 49 & $\begin{array}{l}\text { Speech } \\
\text { therapist }\end{array}$ & & & Medical office & $25^{\prime}$ \\
\hline Volunteer & Theoretical & V2 & Male & 72 & Volunteer & & & Association & $65^{\prime}$ \\
\hline $\mathrm{HCP}$ & Theoretical & НСР79 & Female & 38 & $\begin{array}{l}\text { Speech } \\
\text { therapist }\end{array}$ & & & Medical office & $40^{\prime}$ \\
\hline HCP & Theoretical & НCP810 & Female & 52 & $\begin{array}{l}\text { Speech } \\
\text { therapist }\end{array}$ & & & Medical office & $45^{\prime}$ \\
\hline $\mathrm{HCP}$ & Theoretical & НСР911 & Male & 58 & Physician & & & Medical office & $35^{\prime}$ \\
\hline
\end{tabular}


Figure 1 - Theoretical model

No legends 


\section{Figure 1 - Theoretical model}

\section{Persuading the patient of an obligation}

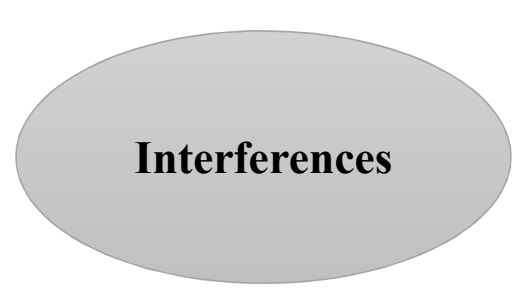

Shock for the diagnosis experienced by the patients

Patients' expectations regarding the functional outcomes of the surgery

Cognitive and educational level of the patients

Professionals who potentially might be "offline"

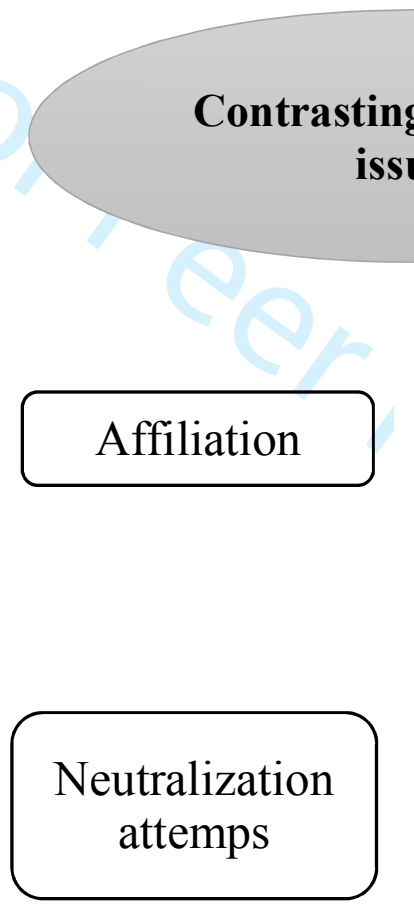

Departmental culture

Shared communication

Feel like a family

Total trust from the patients

Stereotype and stigma

Simplified communication

Information needs suspended

\section{"I will let you}

\section{convince me"}

To save and be saved
The family role
Support team decisions
Emotional needs of patients

